\title{
Intraepithelial Neoplasia in Barrett Esophagus
}

National Cancer Institute

\section{Source}

National Cancer Institute. Intraepithelial Neoplasia in Barrett Esophagus. NCI Thesaurus.

Code C95613.

Glandular intraepithelial neoplasia arising in Barrett esophagus. 